\title{
Is There Benefit from Stenting on Cognitive Function in Intracranial Atherosclerosis?
}

\author{
Tanya N. Turan ${ }^{a}$ Alison Smock ${ }^{a}$ George Cotsonis ${ }^{b}$ David Bachman ${ }^{a}$ \\ Sami Al Kasab ${ }^{a}$ Michael J. Lynn ${ }^{b}$ Azhar Nizham ${ }^{b}$ Collin P. Derdeyn ${ }^{c}$ \\ David Fiorellad $^{d}$ Scott Janis ${ }^{e}$ Bethany Lane ${ }^{b}$ Jean Montgomery ${ }^{b}$ \\ Marc I. Chimowitz ${ }^{a}$ for the SAMMPRIS Investigators \\ ${ }^{a}$ Medical University of South Carolina, Charleston, S.C., ${ }^{\text {b}}$ Emory University, Atlanta, Ga., CUniversity of lowa Hospitals \\ and Clinic, lowa City, lowa, 'State University of New York at Stony Brook, Stony Brook, N.Y., and e National Institute \\ of Neurological Disorders and Stroke, Bethesda, Md., USA
}

\section{Key Words}

Intracranial stenosis · Stroke · Cognition · Revascularization · Angioplasty and stenting

\footnotetext{
Abstract

Background: Revascularization of stenotic cerebral arteries is hypothesized to improve cognition by increasing cerebral perfusion. Aims: We compared cognition impairment among patients treated with percutaneous angioplasty and stenting (PTAS) and aggressive medical management (AMM) versus AMM alone in the Stenting versus Aggressive Medical Therapy for Intracranial Arterial Stenosis (SAMMPRIS) Trial. Methods: In SAMMPRIS, 451 patients with recent transient ischemic attack or stroke attributed to 70-99\% intracranial stenosis were randomized to PTAS plus AMM or AMM alone. Patients who had stroke as the qualifying event with National Institutes of Health Stroke Scale indicating aphasia or neglect were excluded from these analyses. Patients with a cerebrovascular event (ischemic stroke, cerebral infarct with
}

temporary signs or intracranial hemorrhage) during followup were excluded from follow-up visit analyses. The Montreal Cognitive Assessment (MoCA) score was used to assess cognition impairment at baseline, 4 months, 12 months and closeout. Cognitive impairment was defined as MoCA $<26$. Mean MoCA scores and the percentage of patients with cognitive impairment were compared between treatment groups at each time point using t tests and chi-square tests. Differences in MoCA mean at baseline and follow-up time points were compared using mixed model repeated measures ANOVA and Tukey-Kramer tests. Results: There were no significant differences between the treatment groups for mean MoCA at any time point. Mean MoCA scores improved in both groups. The percentage of patients with cognitive impairment in the AMM versus PTAS groups was not significantly different at any time point. Conclusions: Revascularization with PTAS showed no improvement in cognitive impairment over AMM alone among patients who did not have recurrent cerebrovascular events during follow-up.

(c) 2016 S. Karger AG, Basel

\section{KARGER}

(c) 2016 S. Karger AG, Basel

E-Mail karger@karger.com

www.karger.com/ced
Tanya N. Turan, MD, MS

MUSC Stroke Program

19 Hagood Avenue, Suite 501

Harborview Office Tower, Charleston, SC 29425 (USA)

E-Mail turan@musc.edu 


\section{Introduction}

Cognitive function is an important secondary outcome in stroke prevention studies, particularly in trials that study interventions affecting cerebral blood flow. Revascularization procedures of stenotic cerebral or pre-cerebral arteries are of particular interest due to a hypothesized improvement in brain perfusion resulting in preservation or improvement of cognition, independent of stroke outcomes. For this reason, cognitive impairment was a pre-specified secondary outcome in the Stenting versus Aggressive Medical Therapy for Intracranial Arterial Stenosis (SAMMPRIS) trial that studied revascularization of stenotic cerebral arteries. While SAMMPRIS showed that aggressive medical management (AMM) alone was superior to percutaneous angioplasty and stenting (PTAS) plus AMM for secondary stroke prevention [1], we sought to determine if patients treated with PTAS would have less cognitive impairment, independent of stroke recurrence, compared to those treated with AMM alone.

\section{Methods}

The design, participant characteristics and primary outcome results of SAMMPRIS have been previously reported [1-3]. In brief, patients with recent transient ischemic attack (TIA) or stroke attributed to $70-99 \%$ intracranial stenosis were randomized to treatment with PTAS plus AMM or AMM alone. Patients randomized to PTAS were required to undergo PTAS within 3 business days of randomization. In order to minimize the effect of focal neurological deficits from stroke (e.g., aphasia or neglect) on cognitive function, patients presenting with stroke as the qualifying event who had a National Institutes of Health Stroke Scale (NIHSS) score that included points for aphasia or neglect were excluded from these analyses. Similarly, in order to assess the impact of treatment on cognitive function, independent of the treatment's stroke prevention impact, patients with symptomatic cerebrovascular events (ischemic stroke, cerebral infarct with temporary signs or intracerebral hemorrhage) during follow-up were excluded from these analyses. Details regarding the adjudication of events are described elsewhere [2]. In brief, study neurologists who were not masked to treatment assignment evaluated patients with a potential event. Patients with neurological events that were potentially difficult to classify (a TIA lasting $>1 \mathrm{~h}$ or mild ischemic stroke (an increase in the patient's NIHSS score of $<4$ from study entry)) were evaluated by a second neurologist who was masked to treatment. Both neurologists' assessments were sent for central adjudication by neurology adjudicators who were masked to treatment assignment.

The Montreal Cognitive Assessment (MoCA) was used to assess cognitive impairment. MoCA is a screening tool that is sensitive to cognitive deficits arising from stroke and vascular cognitive impairment [4]. MoCA was performed at baseline, 4 months,
12 months and closeout. Cognitive impairment was defined as a MoCA score $<26$, the standard value that has $90 \%$ sensitivity for detecting mild cognitive impairment [5]. Additional analyses also examined cognitive impairment using a MoCA $<23$ and mean MoCA scores. We also examined a MoCA subscore of 11 points that focused on frontal and subcortical function (MoCA FS), which used the Trails B, clock drawing, attention (3 tasks) and fluency portions of the MoCA. In addition, the percentage of patients in each treatment group who had a change in MoCA of $\geq 1$ points was also compared for the group overall and the subset of patients with $>3$ years of follow-up.

Mean MoCA scores and the percentage of patients with cognitive impairment, using the MoCA thresholds and FS subscore described above, were compared between treatment groups at each time point using $t$ tests and chi-square tests. The percentage of patients with a change in MoCA of $\geq 1$ point was compared between treatment groups using chi-square tests. Differences in MoCA mean at the baseline and follow-up time points for each treatment group were compared using mixed model repeated measures, ANOVA and Tukey-Kramer tests and adjusted for age, gender, hypertension, diabetes, lipid disorder, old infarcts, stroke as the qualifying event and location of the symptomatic artery.

\section{Results}

Of the 451 patients enrolled in SAMMPRIS, 371 had MoCA recorded at baseline and did not have a NIHSS score that indicated aphasia or neglect ( $\mathrm{n}=188$ in AMM group, $\mathrm{n}=183$ in PTAS group). At 12 months, 253 of these patients had MoCA data and did not have a cerebrovascular event in the preceding 12 months. At closeout, 199 patients had MoCA data and did not have a cerebrovascular event prior to closeout. Mean follow-up was 3.05 and 3.12 years in the AMM and PTAS groups, respectively. The number of subjects with at least 3 years of follow-up were similar between the AMM $(n=59)$ and PTAS $(\mathrm{n}=54)$ groups.

The percentage of patients with cognitive impairment $(\mathrm{MoCA}<26)$ in the AMM versus PTAS groups was not significantly different at baseline ( 53 vs. $56 \%, p=0.55$ ), 12 months ( 42 vs. $40 \%, \mathrm{p}=0.70$ ) and closeout ( 43 vs. $38 \%$, $\mathrm{p}=0.48$ ). Similarly, at both 12 months and closeout, cognitive function was not impacted by treatment when using a MoCA cutoff of $<23$ ( $\mathrm{p}=0.94$ and $\mathrm{p}=0.81$, respectively). Mean MoCA scores were not different between AMM versus PTAS at 12 months $(25.4 \pm 3.73$ vs. $25.65 \pm$ $3.89, \mathrm{p}=0.55)$ and closeout $(25.58 \pm 3.61$ vs. $25.66 \pm 3.76$, $\mathrm{p}=0.90)$. There was also no difference in mean MoCA FS at 12 months $(p=0.93)$ and closeout $(p=0.69)$. The percentage of patients in each treatment group who had an increase or decrease in MoCA score from baseline to closeout was not different for the group overall $(\mathrm{p}=0.59)$
Turan et al. 
Fig. 1. MoCA score by treatment assignment during follow-up. ${ }^{*} \mathrm{p}<0.05$ for change from baseline.

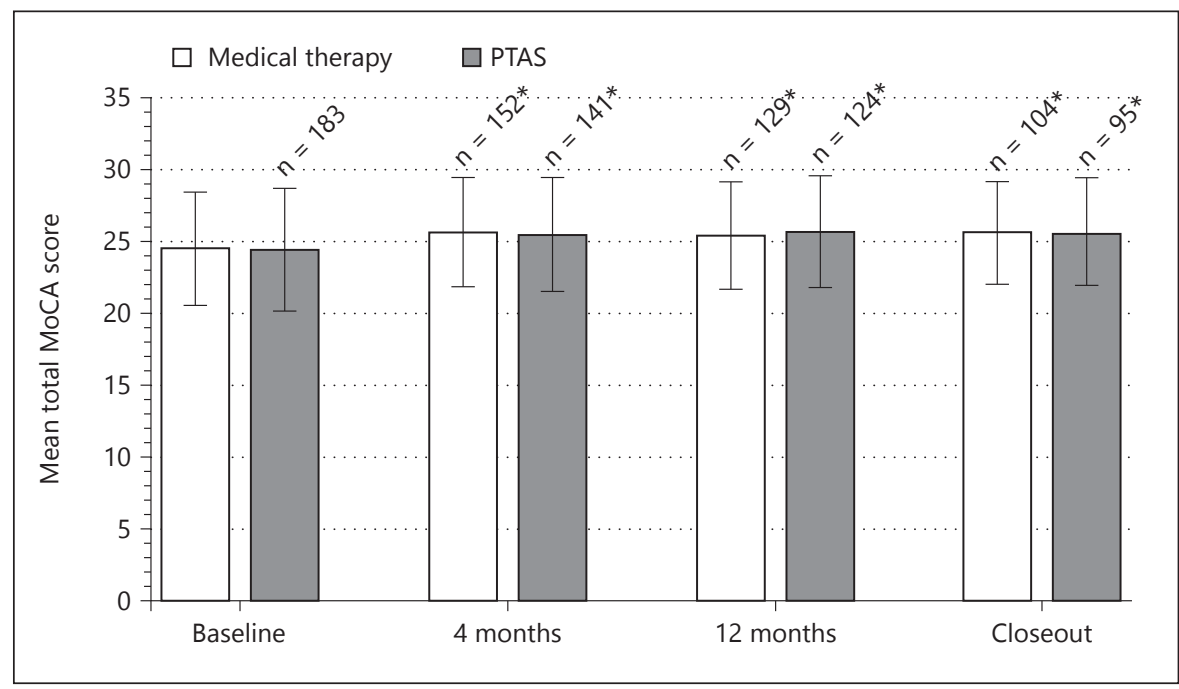

Table 1. Change in MoCA score among SAMMPRIS patients by treatment assignment

\begin{tabular}{lll}
\hline $\begin{array}{l}\text { Change in MoCA } \\
\text { points }\end{array}$ & $\begin{array}{l}\text { AMM (medical } \\
\text { therapy only), n (\%) }\end{array}$ & $\begin{array}{l}\text { PTAS (stenting and } \\
\text { medical therapy), n (\%) }\end{array}$ \\
\hline $\begin{array}{l}\text { Increase } \geq 1 \\
\text { No change }\end{array}$ & $48(37.5)$ & $39(32.0)$ \\
Decrease $\geq 1$ & $61(47.7)$ & $20(16.4)$ \\
\hline
\end{tabular}

Chi-square $\mathrm{p}$ value $=0.655$.

or for only subjects with $>3$ years of follow-up $(\mathrm{p}=0.65)$, as shown in tables 1 and 2. Patients in both treatment groups had a statistically significant $(\mathrm{p}<0.05)$ approximately 1-point increase in MoCA score from baseline to the follow-up visits, as shown in figure 1. Treatment assignment was not associated with a change in MoCA score from baseline to 1 year or closeout ( $p=0.4747$ and $\mathrm{p}=0.4706$, respectively), even after adjusting for age, gender, hypertension, diabetes, lipid disorder, old infarcts, stroke as the qualifying event and location of the symptomatic artery $(\mathrm{p}=0.4656$ and $\mathrm{p}=0.5640$, respectively).

\section{Discussion}

In SAMMPRIS, revascularization with PTAS did not provide improvement in cognitive assessment scores over AMM alone among patients who did not have recurrent cerebrovascular events during follow-up. While it has been hypothesized that reduced perfusion through a
Table 2. Change in MoCA score among SAMMPRIS patients with $>3$ years of follow-up by treatment assignment

\begin{tabular}{lll}
\hline $\begin{array}{l}\text { Change in MoCA } \\
\text { points }\end{array}$ & $\begin{array}{l}\text { AMM (medical } \\
\text { therapy only), } \mathrm{n}(\%)\end{array}$ & $\begin{array}{l}\text { PTAS (stenting and } \\
\text { medical therapy), } \mathrm{n}(\%)\end{array}$ \\
\hline $\begin{array}{l}\text { Increase } \geq 1 \\
\text { No change }\end{array}$ & $20(33.9)$ & $18(33.3)$ \\
Decrease $\geq 1$ & $32(11.8)$ & $10(18.5)$ \\
\hline
\end{tabular}

Chi-square $\mathrm{p}$ value $=0.596$.

large cerebral artery may lead to cognitive decline and that therefore reperfusion of stenotic cerebral arteries would improve cognition, there is a paucity of data that supports this hypothesis. Few randomized trials studying revascularization in patients with large artery cerebrovascular disease have assessed cognition, and the available data are limited only to extracranial arteries [6-8] and not to intracranial arteries. Both the Asymptomatic Carotid Artery Study (ACAS), which studied carotid endarterectomy versus medical therapy, and the Randomized Evaluation of Carotid Occlusion and Neurocognition (RECON) trial (an ancillary study of the Carotid Occlusion Surgery Study that studied extracranial-intracranial bypass for carotid occlusion) showed no benefit of revascularization on cognition over time $[6,7]$. ACAS reported a non-significant decline in the MMSE in both treatment groups during the 5 years of follow-up [6]. RECON showed no difference in the change in cognitive function over time between medical or revascularization groups during 2 years of follow-up using a battery of 14 neurocognitive 
tests [7]. Together, analyses of these 3 randomized clinical trials provide no support for the hypothesis that revascularization of large cerebral arteries provides any improvement in cognitive assessment scores.

Overall, SAMMPRIS patients in both treatment groups did have a small improvement in performance in MoCA testing during a mean follow-up of 3 years. However, both groups also had a large percentage of patients with at least minimal decline. Given that multimodal risk factor control, including healthy diet, exercise and vascular risk factor medications, improves cognitive outcome in healthy older adults [9], it is possible that the intensive risk factor treatment provided in SAMMPRIS [3] may have resulted in some cognitive improvement in this high-risk stroke population. Future analyses on the impact of risk factor control on MoCA scores in SAMMPRIS may address this issue. However, the increase in MoCA scores seen in both groups during follow-up may also be due to a possible learning effect of the MoCA, as an increase of 0.9 points has been reported at 1 month [5].

A limitation of our study is that we cannot account for the impact of silent cerebral infarcts, which may have been more prevalent in one treatment group, on the MoCA scores because brain imaging was not required in patients without neurological symptoms. Similarly, angiography during follow-up was not permitted; so the impact of restenosis or occlusion on MoCA scores cannot be assessed. The use of MoCA to assess cognitive function may also be considered a limitation. While the MoCA can be sensitive for screening for cognitive impairment, it does not provide the detailed assessment of subtle changes in cognitive function that a more comprehensive neuropsychological evaluation would. Future studies of treatments for symptomatic intracranial stenosis could address this issue by performing more detailed neuropsychiatric testing. However, given that stenting of stenotic intracranial arteries is not recommended for stroke prevention [10], future studies to assess the impact of revascularization on stroke prevention and/or cogni- tion are likely to be limited to very select high-risk patients and not generalizable to all patients with intracranial stenosis.

\section{Summary}

In conclusion, among patients with symptomatic severe intracranial atherosclerotic stenosis, revascularization does not provide any independent benefit on cognitive assessment scores over medical therapy and, therefore, is unwarranted for that purpose.

\section{Funding Source}

SAMMPRIS was funded by the National Institute of Neurological Disorders and Stroke (NINDS) U01 NS058728.

\section{Disclosure Statement}

T.N.T., C.P.D., D.F., M.I.C., G.C., M.J.L., A.N., B.L. and J.M. received funding from NIH/NINDS during the conduct of SAMMPRIS. T.N.T. received funding a K23 grant from NIH/ NINDS, and Gore and Associates and Boehringer Ingelheim (Clinical Events Adjudicator) unrelated to this work. A.S., S.A.K., S.J. and D.B. have no disclosures. C.P.D. has relationships with companies that manufacture medical devices for the treatment of cerebrovascular disease, unrelated to this work: W.L. Gore and Associates (Scientific Advisory Board and Consultant); Microvention, Inc. (Angiographic Core Lab for clinical trial); Penumbra, Inc. (DSMB member); Pulse Therapeutics (Chair, Scientific Advisory Board). D.F. received research/salary support from Siemens, Microintervention, Penumbra and Sequent; consulting fees from Covidien/Ev3, Codman and Shurtleff, Penumbra, Microvention; royalties from Codman and Shurtleff (REVIVE); and Vascular Simulators LLC. M.I.C. received other grants from NIH/NINDS related to the treatment of intracranial arterial stenosis; support from Astra Zeneca and Stryker Neurovascular (formerly Boston Scientific Neurovascular) related to the SAMMPRIS trial; Gore Associates (DSMB member); Merck/Parexel and Medtronic (Clinical Trial Event Adjudicator) unrelated to the submitted work; personal fees as an Expert Witness in medical legal stroke cases.

\section{References}

1 Derdeyn CP, Chimowitz MI, Lynn MJ, Fiorella D, Turan TN, Janis LS, et al: Aggressive medical treatment with or without stenting in high-risk patients with intracranial artery stenosis (SAMMPRIS): the final results of a randomised trial. Lancet 2014;383:333-341.

2 Chimowitz MI, Lynn MJ, Turan TN, Fiorella $\mathrm{D}$, Lane BF, Janis S, et al: Design of the stent- ing and aggressive medical management for preventing recurrent stroke in intracranial stenosis trial. J Stroke Cerebrovasc Dis 2011; 20:357-368.

3 Turan TN, Lynn MJ, Nizam A, Lane B, Egan $\mathrm{BM}$, Le NA, et al: Rationale, design, and implementation of aggressive risk factor management in the stenting and aggressive medi- cal management for prevention of recurrent stroke in intracranial stenosis (SAMMPRIS) trial. Circ Cardiovasc Qual Outcomes 2012; 5:e51-e60.

4 Popović IM, Serić V, Demarin V: Mild cognitive impairment in symptomatic and asymptomatic cerebrovascular disease. J Neurol Sci 2007;257:185-193. 
5 Nasreddine ZS, Phillips NA, Bédirian V, Charbonneau S, Whitehead V, Collin I, et al: The Montreal Cognitive Assessment, MoCA: a brief screening tool for mild cognitive impairment. J Am Geriatr Soc 2005;53:695-699.

6 Pettigrew LC, Thomas N, Howard VJ, Veltkamp R, Toole JF: Low mini-mental status predicts mortality in asymptomatic carotid arterial stenosis. Asymptomatic Carotid Atherosclerosis Study investigators. Neurology 2000;55:30-34.

7 Marshall RS, Festa JR, Cheung YK, Pavol MA, Derdeyn CP, Clarke WR, et al: Randomized evaluation of carotid occlusion and neurocognition (RECON) trial: main results. Neurology 2014;82:744-751.

8 Witt K, Börsch K, Daniels C, Walluscheck K, Alfke K, Jansen O, et al: Neuropsychological consequences of endarterectomy and endovascular angioplasty with stent placement for treatment of symptomatic carotid stenosis: a prospective randomised study. J Neurol 2007; 254:1524-1532.

9 Ngandu T, Lehtisalo J, Solomon A, Levälahti E, Ahtiluoto S, Antikainen R, et al: A 2 year multidomain intervention of diet, exercise, cognitive training, and vascular risk monitoring versus control to prevent cognitive decline in at-risk elderly people (FINGER): a randomised controlled trial. Lancet 2015;385: 2255-2263.

10 Kernan WN, Ovbiagele B, Black HR, Bravata DM, Chimowitz MI, Ezekowitz MD, et al: Guidelines for the prevention of stroke in patients with stroke and transient ischemic attack: a guideline for healthcare professionals from the American Heart Association/American Stroke Association. Stroke 2014;45: 2160-2236 